\title{
Quinolonas. Perspectivas actuales y mecanismos de resistencia
}

\author{
Diego Abelardo Álvarez-Hernández, Gilda Sofía Garza-Mayén y Rosalino Vázquez-López
}

\section{Quinolones. Nowadays perspectives and mechanisms of resistance}

Quinolones are a family of synthetic broad-spectrum antimicrobial drugs whose target is the synthesis of DNA. They directly inhibit DNA replication by interacting with two enzymes; DNA gyrase and topoisomerase IV. They have been widely used for the treatment of several community and hospital acquired infections, in the food processing industry and in the agricultural field, making the increasing incidence of quinolone resistance a frequent problem associated with constant exposition to diverse microorganisms. Resistance may be achieved by three non-exclusive mechanisms; through chromosomic mutations in the Quinolone Resistance-Determining Regions of DNA gyrase and topoisomerase IV, by reducing the intracytoplasmic concentrations of quinolones actively or passively and by Plasmid-Mediated Quinolones-Resistance genes, [Qnr determinant genes of resistance to quinolones, variant gene of the aminoglycoside acetyltransferase $\left.\left(A A C\left(6^{\prime}\right)-I b-c\right)\right]$ and encoding genes of efflux pumps (qepA and oqxAB)]. The future of quinolones is uncertain, however, meanwhile they continue to be used in an irrational way, increasing resistance to quinolones should remain as an area of primary priority for research.

Key words: Quinolones, fluoroquinolones, antimicrobial drug resistance, bacterial infections, DNA gyrase, topoisomerase IV.

Palabras clave: Quinolonas, fluoroquinolonas, resistencia antimicrobiana a fármacos, infecciones bacterianas, ADN girasa, topoisomerasa IV.

\section{Introducción}

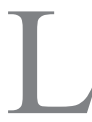

as quinolonas son un grupo de antimicrobianos sintéticos de amplio espectro, cuyo "blanco" es la síntesis del ADN. Se han utilizado ampliamente para el tratamiento de infecciones intra y extra-hospitalarias, convirtiéndose en un recurso de suma importancia para países en vías de desarrollo debido a la gran disponibilidad de genéricos que reducen drásticamente el costo del tratamiento ${ }^{1}$. Su efectividad se debe a su alta biodisponibilidad, nivel de seguridad y forma de administración que puede ser tanto enteral como parenteral ${ }^{2}$. Sin embargo, se han usado indiscriminadamente en el campo de la agricultura y en el procesamiento de alimentos, lo que hace que el incremento de resistencia a quinolonas sea un problema cada vez más frecuente asociado a la constante exposición de diversos microorganismos ${ }^{3,4}$.

\section{Antecedentes históricos y propiedades químicas}

El término "quinolona" se usa en un sentido genérico para referirse a la clase de inhibidores de la síntesis del ADN que incluyen; naftiridonas, quinolonas, isotiazol quinolonas, quinazolinas y agentes relacionados ${ }^{5}$.

El cribado de análogos sintéticos de quinina en búsqueda de nuevos fármacos antimaláricos, condujo al descubrimiento de la 7-cloroquinolina. La investigación de compuestos similares, como 1,8 naftiridonas, resultó en el descubrimiento del ácido nalidíxico (1-etil-7-metil4-oxo-1,8-naftiridina-3-ácido carboxílico) en 1962, convirtiéndose en la primera quinolona sintética con actividad antimicrobiana, especialmente para el tratamiento de infecciones de vías urinarias no complicadas ${ }^{6}$. Esto llevó al desarrollo de otros antimicrobianos basados en el anillo 4-quinolona, como el ácido oxolínico, cinoxacina y flumequina, usados clínicamente para tratar infecciones causadas por bacterias gramnegativas ${ }^{7-9}$.

A través de los años, el ácido nalidíxico ha sido modificado con el objetivo de obtener un espectro más amplio de cobertura antimicrobiana, dando origen a las primeras fluoroquinolonas. Éstas poseen un átomo de flúor unido al anillo de quinolona en la posición C-6, mejorando la actividad contra bacterias gramnegativas y grampositivas ${ }^{10}$. La tercera generación presentó sustituciones de piperazina o pirrolidina en la posición C-7 y de un grupo metoxi en la posición C-8, mejorando la actividad contra bacterias grampositivas y anaerobias. Posteriormente, la cuarta generación presentó múltiples cambios en búsqueda de la mejor combinación entre seguridad y eficacia. Dichos cambios incluyeron la adición de ciclopropil en la posición C-1 y un grupo metoxi en C-8, además, se substituyó el átomo de flúor en C-6 y piperazina o pirrolidina en C-7, por lo que se les nombró des-fluoroquinolonas ${ }^{11,12}$. Existen autores que consideran una quinta generación,
Universidad Anáhuac México Norte.

Facultad de Ciencias de la Salud.

Los autores declaran no tener conflicto de interés alguno. La elaboración del manuscrito no requirió financiamiento alguno.

Recibido: 29 de abril de 2015 Aceptado: 28 de julio de 2015

Correspondencia a: Rosalino Vázquez-López vazquezrosalino@yahoo.com 
con delafloxacina como el único fármaco perteneciente a este grupo.

\section{Clasificación y espectro de actividad}

De acuerdo a su espectro de actividad, las quinolonas se clasifican dentro de cuatro distintas generaciones (Tabla 1).

\section{Mecanismo de acción}

El blanco específico de las quinolonas es interferir en la síntesis del ADN, conduciendo a muerte celular bacteriana mediante la fragmentación cromosómica ${ }^{13}$. Penetran la pared celular a través de porinas, inhibiendo directamente la replicación bacteriana al interactuar con dos enzimas; ADN girasa (proteína tetramérica compuesta por dos pares de subunidades A y B, codificadas por los genes $G y r A$ y $G y r B)^{14}$ y topoisomerasa IV (proteína tetramérica compuesta por dos pares de subunidades A y B, codificados por los genes ParC y ParE $)^{15}$, las cuales son necesarias para realizar el superenrollamiento del ADN. Específicamente, ADN girasa es el blanco primario en bacterias gramnegativas, mientras que topoisomerasa IV lo es en bacterias grampositivas. Algunas quinolonas con espectro de actividad y potencia mejorada, parecen tener como blanco ambas enzimas ${ }^{16}$.

\section{Farmacocinética y farmacodinamia}

La mayoría de las quinolonas se encuentran disponibles de forma oral e intravenosa. La biodisponibilidad, sin importar el modo de administración, es la misma (70$90 \%)^{17}$, aunque la administración vía oral suele verse afectada por la ingesta simultánea de alimentos, antiácidos que contengan magnesio $(\mathrm{Mg})$, aluminio $(\mathrm{Al})$, calcio $(\mathrm{Ca})$ y/o sucralfato, así como multivitamínicos que contengan hierro $(\mathrm{Fe})$ y/o zinc $(\mathrm{Zn})$. En adultos tienen una vida media de 1,5 a 16 h y volúmenes de distribución mayores a 1,5 L/ $\mathrm{kg}^{17-19}$, siendo las de cuarta generación las que alcanzan los valores más altos. Son metabolizadas en el hígado por medio del citocromo p450, aunque algunas también pueden ser metabolizadas por la glucoronidasa o por conjugación de sulfatos ${ }^{20}$. Todas las quinolonas se eliminan vía hepática o renal, las hidrofílicas como levofloxacina y ofloxacina se eliminan por el riñón mientras que las lipofílicas como esparfloxacina, gatifloxacina y moxifloxacina lo hacen a través del hígado ${ }^{21}$.

En proporciones por encima de la concentración inhibitoria mínima (CIM), todas las fluoroquinolonas exhiben tanto efecto post-antibiótico como actividad bactericida dependiente de concentración. Además de actuar sobre la ADN girasa y topoisomerasa IV, algunas fluoroquinolonas como ciprofloxacina y fleoroxacina también actúan en la membrana celular bacteriana ${ }^{20}$, desintegrando las membranas interna y externa, cuando se encuentran en altas concentraciones. Hipotéticamente lo logran a través de la acción de agentes quelantes que remueven cationes, predominantemente iones de Mg. Esto conlleva un incremento de $\sim 35 \%$ en la hidrofobicidad celular y en la sensibilidad hacia la lisis mediada por dodecilsulfato sódico y otros detergentes ${ }^{22}$.

\section{Tabla 1. Generaciones de quinolonas, representantes y espectro de actividad}

\section{Generación Representantes}

$1^{\mathrm{a}}$

Ácido nalidíxico, ácido oxolínico, ácido piromídico, ácido pipemídico, rosoxacina, cinoxacina, flumequina

$2^{a}$ Ciprofloxacina, enoxacina, fleroxacina, lomefloxacina, nadifloxacina, norfloxacina, ofloxacina, pefloxacina, rufloxacina

\section{$3^{a}$ \\ Levofloxacina, esparfloxacina, tosufloxacina, temafloxacina,} grepafloxacina

\section{Espectro de actividad}

Principalmente enterobacterias

Bacilos gramnegativos: E. coli, Proteus spp., Klebsiella spp., Enterobacter spp., Serratia spp., Citrobacter spp., Salmonella spp., Shigella spp.

Mismas que $1^{\text {a }}$ generación. Espectro extendido en bacilos gramnegativos, inicia cobertura contra cocáceas grampositivas y "atípicos"

Bacilos gramnegativos: P. aeruginosa, M. catarrhalis, Acinetobacter spp., S. maltophilia,

$H$. influenzae, N. gonorrhoeae, V. cholerae, Campylobacter spp.

Cocáceas grampositivas: S. aureus, S. epidermidis

Patógenos atípicos: C. trachomatis, Mycoplasma spp.

Mismas que la $2^{a}$ generación. Bacilos gramnegativos, espectro extendido en cocáceas grampositivas y "atípicos"

Bacilos gramnegativos: Mismos que en $1^{\text {a }}$ y $2^{\mathrm{a}}$ generación

Cocáceas grampositivas: S. pneumoniae y $S$. pyogenes

Patógenos atípicos: C. pneumoniae y $M$. pneumoniae

$4^{\mathrm{a}} \quad$ Balofloxacina, clinafloxacina, gatifloxacina, gemifloxacina, moxifloxacina, pazufloxacina, arenfloxacina, sitafloxacina, rovafloxacina, trovafloxacina
Mismas que la $3^{a}$ generación. Bacilos grampositivos, espectro extendido en anaerobios y "atípicos" Cocáceas grampositivas: S. pneumoniae, S. aureus (resistente a penicilina)

Anaerobios: Bacteroides spp., Clostridium spp.

Patógenos atípicos: L. pneumophila, C. pneumoniae, M. pneumoniae, Ureaplasma spp. 


\section{Aplicaciones clínicas}

Entre las indicaciones para el uso de quinolonas se encuentran las siguientes; infecciones del tracto urinario (ITU) no complicadas (principalmente cuando existe resistencia contra el tratamiento de primera línea), ITU complicadas, prostatitis bacteriana crónica, infecciones de transmisión sexual (ITS) e infecciones pélvicas. También se ha documentado su eficacia para el manejo de infecciones cutáneas, osteo-articulares y del tracto gastrointestinal, como quimioprofilaxis en pacientes neutropénicos, post-quirúrgicos y cirróticos con riesgo de desarrollar peritonitis bacteriana espontánea ${ }^{23,24}$. Además, son consideradas como alternativa a los agentes de primera línea para el manejo de infecciones bacterianas del tracto respiratorio, como sinusitis, neumonía adquirida en la comunidad y bronquitis crónica complicada en pacientes inmunocomprometidos ${ }^{25}$.

\section{Efectos adversos}

El uso de quinolonas conlleva una serie de efectos adversos, dentro de los cuales la mayoría son leves pero frecuentes, mientras que otros son inusuales pero graves y han causado el retiro de múltiples fármacos.

Se puede presentar afección gastrointestinal (náusea, vómito y diarrea en 2 a $10 \%$ de los casos) y dérmica (fototoxicidad, eritema, prurito y urticaria en 2,5 a 10\%) como las reacciones adversas más frecuentes, aunque también se han reportado condrotoxicidad $(1,5 \%$, pefloxacina $14 \%)$, tendinitis $(0,4 \%$, pefloxacina $2,7 \%)$, afección en el sistema nervioso central $(0,2$ a $2 \%)$, cardiovascular $(2,9 \%)$, hematológico $(0,02 \%)$ y hepático $(2$ a $3 \%$, grepafloxacina 12 a $16 \%$ ), dependiendo de las propiedades del agente y de las características del paciente ${ }^{11}$. Dentro de dichas propiedades y características, se ha asociado el uso de esparfloxacina y grepafloxacina con alargamiento del segmento QTc $(3 \%)^{26}$; la administración conjunta con anti-inflamatorios no esteroideos (AINE's) al desarrollo de toxicidad ocular (perforaciones corneales, neuropatía óptica y hemorragias de retina en $3,3 \%)^{22,27}$; el uso de levofloxacina y ciprofloxacina con la aparición de diarrea asociada a Clostridium difficile $(2,2 \%)^{28}$; la administración de moxifloxacina y ciprofloxacina a reacciones de hipersensibilidad inmediata $(<1 \%)^{29} \mathrm{y}$ además, los pacientes con condiciones médicas pre-existentes o que se encuentren con tratamiento concomitante pueden tener mayor riesgo de presentar reacciones adversas neuropsiquiátricas ${ }^{30}$.

\section{Perspectivas actuales}

La resistencia hacia antimicrobianos es una de las mayores preocupaciones que existen en medicina en todo el mundo, con patógenos altamente resistentes de múltiples especies difíciles de tratar. Incluso con las guías actuales, cuyo objetivo es preservar la eficacia de los antimicrobianos, la resistencia a quinolonas sigue ocurriendo en rangos que se incrementa en numerosas especies bacterianas y su uso varía alrededor del mundo. Grecia es el mayor usuario de quinolonas y tiene la mayor incidencia de aislados de Escherichia coli resistente a quinolonas, mientras que Suecia tiene la menor tasa de consumo y la menor incidencia de resistencia ${ }^{31}$. En Estados Unidos de América, un incremento de $40 \%$ en el uso de quinolonas ocasionó que se duplicara la tasa de resistencia hacia ciprofloxacina contra bacilos gramnegativos aislados en Unidades de Cuidados Intensivos. En España, el incremento de resistencia a quinolonas ha tenido como resultado que se evite su uso como tratamiento de primera línea para las ITU desde la década de 1990's. En África, India y Vietnam, se ha observado un incremento en la resistencia hacia quinolonas para el tratamiento de ciertas cepas de Salmonella Typhi, mientras que en Finlandia se ha reportado un incremento en la CIM de ciprofloxacina en aislados de Salmonella enterica de viajeros que habían ido hacia el sudeste de $\mathrm{Asia}^{32}$.

También existen diferencias dependiendo de la procedencia de las cepas, de las manifestaciones clínicas y de las co-morbilidades del paciente. Por ejemplo; las tasas de resistencia hacia quinolonas van desde 2,2 a $69 \%$ para cepas aisladas de pacientes con ITUs no complicadas adquiridas en la comunidad y hasta $98 \%$ para cepas de pacientes con ITUs complicadas. Respecto a las ITUs adquiridas en el hospital, la resistencia hacia quinolonas va de 6,3 a $62 \%$ en cepas gramnegativas, $20 \%$ en $S$. aureus sensible a meticilina (SASM) y $100 \%$ en $S$. aureus resistente a meticilina (SARM). En general, los uropatógenos provenientes de pacientes admitidos en hospitales son menos sensibles al uso de quinolonas que las de aquellos que se encuentran en la comunidad y aunque la resistencia hacia quinolonas en Norte América es menos frecuente que en Asia y Europa, las elevadas tasas de migración convierten a la región en una zona altamente vulnerable, $y$, por lo tanto, el conocimiento de los mecanismos mediante los cuales éstas se desarrollan adquiere relevancia ${ }^{33}$.

\section{Mecanismos de resistencia}

La resistencia puede alcanzarse mediante tres mecanismos no exclusivos:

- A través de mutaciones cromosómicas en genes codificantes, al reducir las concentraciones intracitoplásmicas de quinolonas y por genes de resistencia a quinolonas mediados por plásmidos (PMQR, por sus siglas en inglés). 
- Las mutaciones cromosómicas afectan las regiones determinantes de resistencia a quinolonas (QRDR, por sus siglas en inglés) de ADN girasa (GyrA y GyrB) y topoisomerasa IV (ParC y ParE) $)^{3}$. Se dan por errores de transcripción durante la replicación cromosómica y ocurren en rangos tan altos como 1 en $10^{6}$ a $10^{9}$ en bacterias silvestres ${ }^{34}$. Las mutaciones en gyrA, el gen que codifica las subunidades A de ADN girasa, conforman el mecanismo que con mayor frecuencia se encuentra involucrado en la resistencia a quinolonas entre las bacterias gramnegativas ${ }^{35}$.

- La reducción de la concentración intracelular de quinolonas se da principalmente por dos mecanismos; pasivamente, mediante reducción de la permeabilidad por "downregulation" de proteínas extra-membranales que forman canales y activamente, mediante sobreexpresión de sistemas de eflujo multidroga pertenecientes a la superfamilia de división de resistencia-nodulación $(\mathrm{DRN}), \mathrm{Acr} A \mathrm{~B}-\mathrm{TolC}^{36}$. Estos últimos reducen la acumulación de quinolonas en el citoplasma, proporcionando el tiempo suficiente para que la bacteria se adapte y adquiera resistencia ${ }^{37}$.

- Actualmente se encuentran descritos tres tipos de genes de resistencia a quinolonas mediados por plásmidos (PMQR), cuyas variaciones se han reportado en diferentes patógenos alrededor del mundo; genes de resistencia a quinolonas determinates de $q n r$, gen variante de la aminoglucósido acetil transferasa $\left(A A C\left(6^{\prime}\right)-l b-c r\right)$ y genes codificadores de bombas de eflujo (qepA y oqx $A B)^{4,38,39}$.

a) Los $q n r$, son genes transferibles que confieren baja resistencia, tanto a quinolonas como a fluoroquinolonas. Se han identificado con claridad cinco familias: $q n r A, q n r B, q n r C, q n r D$ y $q n r S^{40,41}$; sin embargo, en 2013, Pons y cols., describieron una sexta familia de $q n r ' s$ : los $q n r C V^{42}$. Estos genes codifican para la formación de una proteína de 218 aminoácidos de repetición de pentapéptidos, la cual protege a ADN girasa y a topoisomerasa IV de la inhibición de las quinolonas mediante la reducción de la disponibilidad de los blancos holoenzima de $\mathrm{ADN}^{43-45}$. La presencia de genes qnr puede aumentar el número de mutaciones que causan resistencia a quinolonas; además, suelen combinarse con otros genes dentro del mismo plásmido, generando resistencia cruzada entre quinolonas y otros antimicrobianos a los que no se era previamente resistente ${ }^{39}$.

b) En 2006, Robicsek y cols., describieron, por primera vez, el gen $a a c\left(6^{\prime}\right)-I b-c r$, una nueva variante de la aminoglucósido acetiltransferasa o $\mathrm{Aac}\left(6^{\prime}\right)-\mathrm{Ib}$, responsable de la resistencia de cepas de enterobacterias hacia amikacina, kanamicina y tobramicina. Este gen produce una $\mathrm{N}$-acetilación en el sustituto de piperazinil en algunas fluoroquinolonas, incluyendo norfloxacina y ciprofloxacina, lo que reduce su actividad antimicrobiana ${ }^{45}$. La enzima $\mathrm{Aac}\left(6^{\prime}\right)-\mathrm{Ib}-\mathrm{cr}$ se distingue de $A a c\left(6^{\prime}\right)-I b$ por la presencia de dos mutaciones puntuales en la misma; Trp102 $\rightarrow$ Arg y Asp 179 $\rightarrow T y r^{46}$, ambas responsables de la reducción de la susceptibilidad (dos a tres veces mayor) de algunas fluoroquinolonas, sumando este efecto a otros mecanismos codificados por plásmidos ${ }^{45}$. En 2013, Silva-Sánchez y otros concluyeron que la prevalencia de genes PMQR ( $q n r$, aac (6')-Ib-cr y qepA) en cepas de enterobacterias (K. pneumoniae, E. coli, E. cloacae) en pacientes pediátricos en México era de $32,1 \%{ }^{47}$.

c) El gen qepA codifica para generar una bomba de eflujo tipo QepA que confiere resistencia a quinolonas hidrofílicas como norfloxacina, ciprofloxacina y enrofloxacina por medio del sistema similar a la súperfamilia de bombas de eflujo tipo facilitador mayor (MFS, por sus siglas en inglés). Este gen se identificó en plásmidos pHPA de $E$. coli $\mathrm{C} 316$ y en el plásmido pIP1206 de $E$. coli 1450 , aislados en Japón y en Bélgica, respectivamente ${ }^{48,49}$. Así mismo, OqxAB también es un plásmido codificador para bombas de eflujo, siendo identificado recientemente como uno de los PMQR. OqxAB es codificado por los genes $o q x A$ y $o q x B$, localizados en un plásmido de $52 \mathrm{~kb}$ designado pOLA52 que confiere resistencia para múltiples agentes, incluyendo las fluoroquinolonas $^{50}$. En comparación con otros mecanismos de resistencia mediados por plásmidos, se conoce muy poco sobre la prevalencia de OqxAB y su contribución con la resistencia a diferentes antimicrobianos.

En la Tabla 2 se resumen los principales mecanismos de resistencia a quinolonas.

\section{Conclusiones}

La velocidad con que se desarrollan nuevos fármacos se encuentra por debajo de aquella con la que se desarrollan nuevas resistencias, haciendo que el futuro de las quinolonas sea incierto. Por lo tanto, el uso indiscriminado de antimicrobianos es una práctica que atañe al interés de toda la comunidad científica, puesto que los microorganismos adquieren y modifican constantemente sus mecanismos de defensa y el incremento de resistencias merma cada vez más nuestros recursos terapéuticos para el manejo de infecciones en el ser humano. Es necesario concientizar al personal de salud sobre la importancia de realizar investigación en tales áreas y de mejorar el apego hacia las guías de práctica clínica que permitan prolongar la utilidad de las quinolonas y de otros antimicrobianos. 


\begin{tabular}{|c|c|c|c|c|}
\hline Mecanismo & \multicolumn{4}{|l|}{ Características } \\
\hline Mutaciones cromosómicas & \multicolumn{4}{|c|}{$\begin{array}{l}\text { Afectan las regiones determinantes de resistencia a quinolonas de ADN girasa (GyrA y GyrB) y topoisomerasa IV (ParC y ParE). Se dan } \\
\text { por errores de transcripción durante la replicación cromosómica y ocurren en rangos tan altos como } 1 \text { en } 10^{6}-10^{9} \text { en bacterias silvestres }\end{array}$} \\
\hline $\begin{array}{l}\text { Reducción de la concentración } \\
\text { intracelular de quinolonas }\end{array}$ & \multicolumn{2}{|c|}{$\begin{array}{l}\text { Se da principalmente por dos mecanismos: } \\
\text { Pasivamente: Reducen la permeabilidad por "downregulation" } \\
\text { de proteínas extra-membranas que forman canales }\end{array}$} & \multicolumn{2}{|c|}{$\begin{array}{l}\text { Activamente: Sobre-expresan sistemas de eflujo multidroga } \\
\text { pertenecientes a la super-familia de división de resistencia- } \\
\text { nodulación (DRN), AcrAB-TolC }\end{array}$} \\
\hline $\begin{array}{l}\text { Genes de resistencia a quinolonas } \\
\text { mediados por plásmidos }\end{array}$ & $\begin{array}{l}\text { Se da principalmente por tres mecanismos: } \\
\text { Qnr: Son genes transferibles que pueden } \\
\text { aumentar el número de mutaciones que } \\
\text { causen resistencia a quinolonas }\end{array}$ & \multicolumn{2}{|c|}{$\begin{array}{l}\left.\text { Gen aac( } 6^{\prime}\right)-I b \text {-cr: Es una variante de ami- } \\
\left.\text { noglucósido acetiltransferasa o aac( } 6^{\prime}\right)-1 b \\
\text { que reduce la actividad antimicrobiana de } \\
\text { algunas quinolonas }\end{array}$} & $\begin{array}{l}\text { Genes qepA y OqxAB: Codifican para } \\
\text { generar una bomba de eflujo tipo QepA } \\
\text { que confiere resistencia a quinolonas } \\
\text { hidrofílicas }\end{array}$ \\
\hline
\end{tabular}

\section{Resumen}

Las quinolonas son un grupo de antimicrobianos sintéticos de amplio espectro, cuyo objetivo es la síntesis del ADN. Inhiben directamente su replicación al interactuar con dos enzimas; ADN girasa y topoisomerasa IV. Se han utilizado ampliamente para el tratamiento de infecciones intra y extra-hospitalarias, en el campo de la agricultura y en el procesamiento de alimentos, lo que hace que el incremento de resistencia a quinolonas sea un problema cada vez más frecuente, asociado a la constante exposición de diversos microorganismos. La resistencia puede alcanzarse mediante tres mecanismos no excluyentes entre sí; a través de mutaciones cromo- sómicas en genes codificantes que afectan las regiones determinantes de resistencia a quinolonas de ADN girasa y topoisomerasa IV, al reducir las concentraciones intracitoplásmicas de quinolonas de manera activa o pasiva y por genes de resistencia a quinolonas mediados por plásmidos [genes de resistencia a quinolonas determinates de $q n r$, gen variante de la aminoglucósido acetil transferasa (AAC ( $\left.6^{\prime}\right)-l b-c r$ ) y genes codificadores de bombas de eflujo (qepA y oqxAB)]. El futuro de las quinolonas es incierto; sin embargo, mientras continúen empleándose para el manejo de infecciones en el ser humano, el incremento de resistencia a quinolonas debe permanecer como un área de importancia primaria para la investigación.

\section{Referencias bibliográfícas}

1.- Pallecchi L, Bartoloni A, Riccobono E, Fernández C, Mantella A, Magnelli D, et al. Quinolone resistance in absence of selective pressure: the experience of a very remote community in the Amazon Forest. PLoS Negl Trop Dis 2012; 6 (8): e1790.

2.- Mehlhorn A J, Brown D A. Safety concerns with fluoroquinolones. Ann Pharmacother 2007; 41: $1859-66$

3.- Ambrozic A J, Keber R, Zerjavic K, Orazem T, Grabnar M. Emergence of the quinolone resistance-mediating gene $a a c\left(6^{\prime}\right)-I b-c r$ in extended spectrum-beta-lactamase-producing Klebsiella isolates collected in Slovenia between 2000 and 2005. Antimicrob Agents Chemother 2007; 51: 4171-3.

4.- Strahilevitz J, Jacoby G A, Hooper D C, et al. Plasmid-mediated quinolone resistance, a multifaceted threat. Clin Microbiol Rev 2009; 22: 664-89.

5.- Drlica K, Hiasa H, Kerns R. Quinolones: action and resistance updated. Curr Top Med Chem
2009; 9: 981-98.

6.- Ward J F N C, Jichlinski D, Macis R. Nalidixic acid in urinary infections. Br Med J 1963; 2 (5368): 1311-4.

7.- Mardh P A, Colleen S, Andersson K E. Studies on cinoxacin. 1. In vitro activity of cinoxacin, as compared to nalidixic acid, against urinary tract pathogens. J Antimicrob Chemother 1977; 3: 411-6.

8.- $\quad$ Rohlfing S R, Gerster J R, Kvam D C. Bioevaluation of antibacterial flumequine for urinary tract use. Antimicrob Agents Chemother 1976; 10: 20-4.

9.- Barry A L, Jones R N, Thornsberry C, Ayers L W, Gerlach E H, Sommers H M. Antibacterial activities of ciprofloxacin, norfloxacin, oxolinic acid, cinoxacin, and nalidixic acid. Antimicrob Agents Chemother 1984; 25: 633-7.

10.- Wiles J A, Bradbury B J, Pucci M J. New quinolone antibiotics: a survey of the literature from 2005 to 2010. Exp Opin Therap Patents 2010; 20 (10): 1295-319.

11.- Van Bambeke F, Michot J M, Van Eldere J,
Tulkens P M. Quinolones in 2005: an update. Clin Microbiol Infect 2005; 11: 256-80.

12.- Oliphant C M, Green G M. Quinolones: a comprehensive review. Am Fam Physician 2002; 65 (3): 455-65.

13.- Lapongov I, Sohi M K, Veselkov D A, Pan X S, Saehney R, Thompson A W, et al. Structural insight into the quinolone-DNA cleavage complex of type IIA topoisomerases. Nat Struct Mol Biol 2009; 16 (6): 667-9.

14.- Maruri F, Sterling T R, Kaiga A W, Blackman A, van der Heijden Y F, Mayer C, et al. A systematic review of gyrase mutations associated with fluoroquinolone-resistant Mycobacterium tuberculosis and a proposed gyrase numbering system. J Antimicrob Chemother 2012; 67 (4): 819-31.

15.- Nitiss J L. DNA topoisomerase II and its growing repertoire of biological functions. Nat Rev Cancer 2009; 9 (5): 327-37.

16.- Blondeau J M. Fluoroquinolones: mechanism of action, classification and development of resistance. Sur Ophtha 2004; 49 (2): S73-8.

17.- De Smet J, Colin P, De Paepe P, Ruige J, 
Batens H, Van Nieuwenhove, et al. Oral bioavility of moxifloxacin after Roux-en-Y gastric bypass surgery. J Antimicrob Chemother 2012; 67: 226-9.

18.- Lewis R J, Mohr J F. Dysglycaemias and fluoroquinolones. Drug Saf 2008; 31 (4): 283-92.

19.- Turnidge J. Pharmacokinetics and pharmacodynamics of fluoroquinolones. Drugs 1999; 58 (2): 29-36.

20.- Segreti J, Jones R N, Bertino J S. Challenges in assessing microbial susceptibility and predicting clinical response to newer-generation fluoroquinolones. J Ocul Pharmacor Ther 2012; 21 (1): 3-11.

21.- Stein G E. Pharmacokinetics and pharmacodynamics of newer fluoroquinolones. Clin Infect Dis 1996; 23 (1): 19-24

22.- Shaw P J, Ganey P E, Roth R A. Idiosyncratic drug-induced liver injury and the role of inflammatory stress with an emphasis on an animal model of trovafloxacin hepatotoxicity. Toxicol Sci 2010; 118 (1): 7-18.

23.- Andriole V T. The quinolones: past, present, and future. Clin Infect Dis 2005; 41 (2): 113-9.

24.- Shankar T, Vishal M, Prachi T. Quinolones in 2011: An update. J Pharm Res 2011; 4 (4): 1294-7.

25.- Mandell L A, Wunderink R G, Anzueto A, Bartlett J G, Campbell G D, Dean N C, et al. Infectious Diseases Society of America/ American Thoracic Society consensus guidelines on the management of communityacquired pneumonia in adults. Clin Infect Dis 2007; 44 (2): 27-72.

26.- Briasoulis A, Agarwal V, Pierce W J. QT Prolongation and torsade de pointes induced by fluoroquinolones: infrequent side effects from commonly used medications. Cardiology 2011; 120 (2): 103-10.

27.- Etminan M, Forooghian F, Brophy J M, Bird S T, Maberley. Oral fluoroquinolones and the risk of retinal detachment. JAMA 2012; 307 (13): 1414-9.

28.- Ozawa T T, Valadez T. Clostridium difficile infection associated with levofloxacin treatment. Tenn Med 2002; 95: 113-5.

29.- Aranda A, Mayorga C, Ariza A, Doña I, Rosado A, Blanca-López N, et al. In vitro evaluation of IgE-mediated hypersensivity reactions to quinolones. Allergy 2011; 66:
247-54.

30.- Tomé A, Filipe A. Review of phychiatric and neurological adverse reactions. Drug Saf 2011; 34 (6): 465-88.

31.- Redgrave L S, Sutton S B, Webber M A, Piddock L J V. Fluoroquinolone resistance: mechanisms, impact on bacteria, and role in evolutionary success. Trends Microbiol 2014; 22 (8): 438-45.

32.- Guan X, Xue X, Liu Y, Wang J, Wang Y, Wang J, et al. Plasmid-mediated quinolone resistance-current knowledge and future perspectives. J Intern Med Res 2013; 4 (1): 20-30.

33.- Dalhoff A. Global fluoroquinolone resistance epidemiology and implications for clinical use. Interdisc Perspect Infect Dis 2012; 1: 1-37.

34.- Sanders C C. Mechanisms responsible for cross-resistance and dichotomous resistance among the quinolones. Clin Infect Dis 2001; 32 (1): S1-8.

35.- Gruger T, Nitiss J L, Maxwell A, Zechiedrich E L, Heisig P, Seeber S, et al. A mutation in Escherichia coli DNA gyrase conferring quinolone resistance results in sensitivity to drugs targeting eukaryotic topoisomerase II. Antimicrob Agents Chemother 2004; 48 (12): 4495-504.

36.- Rodríguez-Martínez J M, Velasco C, García I, Cano M E, Martínez-Martínez L, Pascual A. Mutant prevention concentrations of fluoroquinolones for expressing the plasmidcarried quinolone resistance determinant qnrA. Antimicrob Agents Chemother 2007; 51 (6): 2236-9.

37.- Li X Z, Nikaido H. Efflux-mediated drug resistance in bacteria: an update. Drugs 2009; 69 (12): 1555-623.

38.- Cattoir V, Potrei L, Aubert C, Soussy C J, Nordmann P. Unexpected occurrence of plasmid-mediated quinolone resistance determinants in environmental Aeromonas spp. Emerg Infect Dis 2008; 14 (2): 231-7.

39.- Hernández A, Sánchez M B, Martínez J L. Quinolone resistance: much more than predicted. Front Microbiol 2011; 2: 22.

40.- Cavaco L M, Hasman H, Xia S, Aarestrup F M. QnrD, a novel gene conferring transferable quinolone resistance in Salmonella enterica serovar Kentucky and Bovismorbificans strains of human origin. Antimicrob Agents Ch 2009;
53 (2): 603-8.

41.- Wang M, Guo Q, Xu X, Wang X, Ye X, $\mathrm{Wu} \mathrm{S}$, et al. New plasmid-mediated quinolone resistance gene, $q n r \mathrm{C}$, found in a clinical isolate of Proteus mirabilis. Antimicrob Agents Chemother 2009; 53 (5): 1892-7.

42.- Pons M J, Gomes C, Ruiz J. QnrVC, a new transferible Qnr-like family. Enferm Infecc Microbiol Clin 2013; 31 (3): 191-2.

43.- Tran J H, Jacoby G A. Mechanism of plasmidmediated quinolone resistance. Proc Natl Acad Sci USA 2002; 99 (8): 5638-42.

44.- Tran J H, Jacoby G A, Hooper D C. Interaction of plasmid encoded quinolone resistance protein qnr with Escherichia coli DNA-gyrase. Antimicrob Agents Chemother 2005; 49 (1): 118-25.

45.- Hegde S S, Vetting M W, Roderick S L, Mitchenall L A, Maxwell A, Takiff H E, et al. A fluoroquinolone resistance protein from Mycobacterium tuberculosis that mimics DNA. Science 2005; 308 (5727): 1480-3.

46.- Robicsek A, Strahilevitz J, Jacoby G A, Macielag M, Abbanat D, Park CH, et al. Fluoroquinolone-modifying enzyme: a new adaptation of a common aminoglycoside acetyltransferase. Nat Med 2006b; 12 (1): 83-8.

47.- Silva-Sánchez J, Cruz-Trujillo E, Barrios H, Reyna-Flores F, Sánchez-Pérez A, Garza-Ramos U, et al. Characterization of plasmid-mediated quinolone resistance (PMQR) genes in extended spectrum beta-lactamaseproducing Enterobacteriaceae pediatric clinical isolates in Mexico. PLoS One 2013; 10 (8): e77968.

48.- Yamane K, Wachino J, Suzuki S, Kimura K, Shibata N, Kato H, et al. New plasmid mediated fluoroquinolone efflux pump, QepA, found in an Escherichia coli clinical isolate. Antimicrob Agents Chemother 2007; 51 (9): 3354-60.

49.- Périchon B, Courvalin P, Galimand M. Transferable resistance to aminoglycosides by methylation of G1405 in 16S rRNA and to hydrophilic fluoroquinolones by QepAmediated efflux in Escherichia coli. Antimicrob Agents Chemother 2007; 51 (7): 2464-9.

50.- Hansen L H, Johannesen E, Burmolle M. A plasmid encoded multidrug efflux pump conferring resistance to olaquindox in Escherichia coli. Antimicrob. Agents Chemother 2004; 48 (9): 3332-7. 